\title{
Light-Induced Precession of Magnetization in Ferromagnetic Semiconductor (Ga,Mn)As
}

\author{
N. Tesařová, P. Němec*, E. Rozkotová, F. Trojánek and P. Malý \\ Charles University in Prague, Faculty of Mathematics and Physics \\ Ke Karlovu 3, 12106 Prague, Czech Republic
}

\begin{abstract}
Precession of magnetization induced by laser pulses in (Ga,Mn)As was studied by the pump-and-probe technique. We concentrated on various experimental aspects that can be used to identify a part of the measured magneto-optical signals that provides information about the dynamics of magnetization. We also revealed that that it takes about 80 ps before the quasi-equilibrium precession of magnetization is initiated by the impact of laser pulse.
\end{abstract}

PACS numbers: 75.50.Pp, 75.78.-n, 78.47.J-, 78.47.D-

\section{Introduction}

Diluted magnetic semiconductors, with (Ga,Mn)As as the most studied representative, have attracted a significant attention in recent years mainly due to the carrier mediated ferromagnetism [1]. The impact of a laser pulse on $(\mathrm{Ga}, \mathrm{Mn})$ As leads to the photo-injection of carriers and to the temperature transient increase that in turn induce a precession of magnetization, which can be detected by a time-resolved magneto-optical (MO) spectroscopy [2-4]. However, we have observed recently that the measured MO signal contains not only the information about the ferromagnetically coupled Mn spins, but it is affected also by the laser-induced change of the complex index of refraction [5]. In this paper we concentrate on the identification of a part of the measured MO signal that provides information about the dynamics of magnetization.

\section{Experimental}

The experiments were performed on the $20 \mathrm{~nm}$ thick ferromagnetic layer of $\mathrm{Ga}_{1-x} \mathrm{Mn}_{x}$ As grown on a GaAs (001) substrate by the low-temperature molecular beam epitaxy. Content of Mn, Curie temperature, and hole concentration in the sample are $x \approx 0.03, T_{\mathrm{C}} \approx 77 \mathrm{~K}$, and $p \approx 4.7 \times 10^{20} \mathrm{~cm}^{-3}$, respectively. The easy axis of magnetization lies in the sample plane; the magnetization is oriented close to [010] direction during the experiment. The polarization of the pump pulses was circular (with the helicity controlled by the quarter-wave plate) and the probe pulses were linearly polarized (along the direction of magnetization). The angles of incidence (measured from the normal of the sample surface) for pump and

* corresponding author; e-mail: nemec@karlov.mff.cuni.cz probe beams were $3^{\circ}$ and $9^{\circ}$, respectively. All experiments were done at $15 \mathrm{~K}$ with no external magnetic field applied $\left(\mu_{0} H_{\text {ext }}<0.1 \mathrm{mT}\right)$ with a time resolution of about 200 fs.

\section{Results and discussion}

The impact of pump laser pulses on the sample modifies its magnetic and optical properties and these changes are detected by time-delayed probe pulses. We used the experimental setup where the pump-induced changes of rotation of probe polarization $(\Delta \theta)$ or ellipticity $(\Delta \eta)$ are measured simultaneously with the transient change of the sample reflectivity $\Delta R / R[3]$.

Typical data of $\Delta \theta$ measured for circularly polarized pump pulses are shown in Fig. 1. The data clearly reveal that after $\approx 100$ ps the dynamics of $\Delta \theta$ does not depend on the helicity of the circular polarization. On the other hand, the signal at shorter time delays is strongly polarization dependent. This polarization-sensitive $\Delta \theta$ signal does not depend significantly on temperature (i.e., it is present even above $T_{\mathrm{C}}$ ), orientation of linear polarization of the probe pulses or external magnetic field (not shown here). The absorption of circularly polarized light leads to a photogeneration of spin-polarized carriers [3]. Therefore, we attribute the polarization-sensitive $\Delta \theta$ signal to the dynamics of spin-polarized electrons. The very similar decay of the polarization-sensitive $\Delta \theta$ signal and the sample reflectivity $\Delta R / R$, which is not sensitive to the pump polarization, shows that the dynamics of spin-polarized electrons is dominated by their lifetime (see the inset of Fig. 1). The most important conclusion for the investigation of magnetization dynamics is that this polarization-sensitive signal does not provide direct information about the ferromagnetic order in the sample.

In the following, we will concentrate on the polarization-independent part of the signal, which is de- 


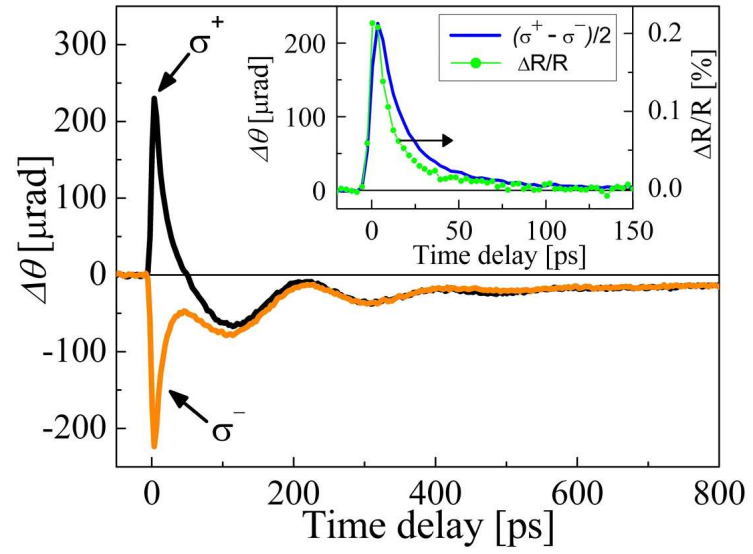

Fig. 1. Dynamics of probe polarization rotation $(\Delta \theta)$ measured for circularly polarized pump pulses $\left(\sigma^{+}\right.$and $\sigma^{-}$) at $15 \mathrm{~K}$ with no external magnetic field applied; $80 \mathrm{fs}$ pulses tuned to $1.63 \mathrm{eV}$ with fluence of $46 \mu \mathrm{J} \mathrm{cm}^{-2}$ were used. Inset: polarization-sensitive part, $\left(\sigma^{+}-\right.$ $\left.\sigma^{-}\right) / 2$, of the measured $\Delta \theta$ signal (solid line) and the dynamics of transient reflectivity (line with points).

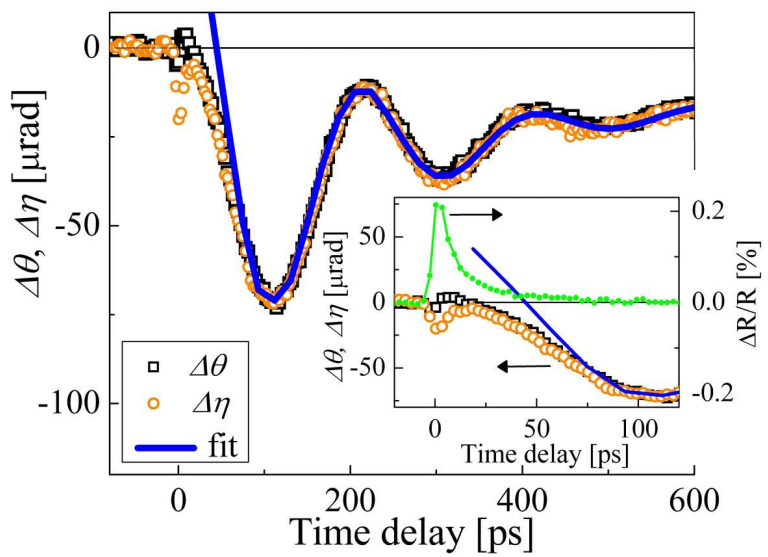

Fig. 2. Polarization-independent part, $\left(\sigma^{+}+\sigma^{-}\right) / 2$, of the rotation $(\Delta \theta)$ and ellipticity $(\Delta \eta)$ (points) measured under identical conditions (we note that no normalization was applied). The solid line is the fit by a sum of the exponentially damped sine harmonic function and the pulse-like function [5]; the parameters of the fit are: $A=82 \mu \mathrm{rad}, \tau_{\mathrm{D}}=145 \mathrm{ps}, \omega=31.3 \mathrm{GHz}, \varphi=59^{\circ}$, $C=-42 \mu \mathrm{rad}, \tau_{1}=1 \mathrm{ps}, \tau_{2}=700 \mathrm{ps}$. Inset: detail of the MO data and the transient reflectivity (line with points).

fined as an average of the signals measured with $\sigma^{+}$and $\sigma^{-}$polarized pump pulses. In Fig. 2 we show the dynamics of $\Delta \theta$ and $\Delta \eta$. The curves are very similar except for short time delays. This implies that in the measured MO signal the "optical part" of the signal [6] has a sizable contribution only in a time range where the reflectivity (i.e., the complex index of refraction) is modified considerably by pump pulses (see the inset of Fig. 2). For time delays larger than $\approx 80$ ps the polarization-independent $\mathrm{MO}$ data can be fitted well by a phenomenological model $[3,5]$ where the signal is decomposed into a damped precession of the magnetization around its quasi-equilibrium easy axis position (the oscillatory signal with amplitude $A$, angular frequency $\omega$, initial phase $\varphi$ and damping time $\left.\tau_{\mathrm{D}}\right)$ and into a tilt of the easy axis in the sample plane (the pulse-like function with amplitude $C$ and rise and decay times $\tau_{1}$ and $\tau_{2}$, respectively). The strong deviation of the fit and the data for shorter time delays (see the inset of Fig. 2) illustrates that it takes about 80 ps to establish a quasi-equilibrium precession of the magnetization. Our observation that the magnetization precession is not fully developed immediately after an impact of the laser pulse is in accord with the results reported also for other magnetic materials. For example, in a very recent experiment in GdFeCo films a time delay of $\approx 90 \mathrm{ps}$ was necessary before the laser pulse-induced switching of magnetization was completed [7].

\section{Conclusions}

We identified a part of the measured MO signal that provides information about the collective oscillations of ferromagnetically coupled Mn spins. We also revealed that the quasi-equilibrium precession of magnetization is initiated as late as 80 ps after the impact of the pump pulse.

\section{Acknowledgments}

We acknowledge V. Novák for sample preparation and characterization and T. Jungwirth for many discussions. This work was supported by Ministry of Education of the Czech Republic (research centre LC510 and the research plan MSM0021620834), Grant Agency of the Czech Republic (grant no. 202/09/H041) and by grant no. SVV-2010-261306 of the Charles University in Prague.

\section{References}

[1] T. Jungwirth, J. Sinova, J. Mašek, A.H. MacDonald, Rev. Mod. Phys. 78, 809 (2006).

[2] A. Oiwa, H. Takechi, H. Munekata, J. Supercond. 18, 9 (2005).

[3] E. Rozkotová, P. Němec, P. Horodyská, D. Sprinzl, F. Trojánek, P. Malý, V. Novák, K. Olejník, M. Cukr, T. Jungwirth, Appl. Phys. Lett. 92, 122507 (2008).

[4] Y. Hashimoto, S. Kabayashi, H. Munekata, Phys. Rev. Lett. 100, 067202 (2008).

[5] E. Rozkotová, P. Němec, N. Tesařová, P. Malý, V. Novák, K. Olejník, M. Cukr, T. Jungwirth, Appl. Phys. Lett. 93, 232505 (2008).

[6] E. Kojima, R. Shimano, Y. Hashimoto, S. Katsumoto, M. Kuwata-Gonokami, Phys. Rev. B 68, 193203 (2003).

[7] K. Vahaplar, A.M. Kalashnikova, A.V. Kimel, D. Hinzke, U. Nowak, R. Chantrell, A. Tsukamoto, A. Itoh, A. Kirilyuk, Th. Rasing, Phys. Rev. Lett. 103, 117201 (2009). 